\title{
Benefits of MR Diffusion Tensor Imaging and Fiber Tractography Over Conventional MRI in Preoperative Evaluation of Pediatric Brain Tumors
}

\author{
SAMIA Y. HEIKAL, M.Sc.*; IMAN M. ZAKY, M.D.*; MOHAMED A. EL-BELTAGY, M.D.** and \\ AYMAN ABD EL-HAMID EL-BASMY, M.D.***
}

The Departments of Radio-diagnosis*, National Cancer Institute, Radiology** and Neurosurgery***, Faculty of Medicine, Cairo University

\begin{abstract}
Background: Diffusion tensor imaging (DTI) is a noninvasive magnetic resonance imaging (MRI) modality which accurately shows the location and orientation of subcortical white matter (WM) tracts. The surgeon benefits from DTI as it depicts the relationship of the intra-axial tumor to local WM tracts in multiple planes.
\end{abstract}

Aim of Study : The purpose of this study is to evaluate the role of MR diffusion tensor imaging and fiber tractography and its benefits over conventional MRI in accurate preoperative localization of important eloquent areas in pediatric brain tissue and their relation to the brain tumor. The aim of the study is to help the surgeon in planning his surgical corridor which causes the least damage and minimizes the postoperative neurological deficits.

Patients and Methods: A total of fifty patients with brain tumors were included in this study using pre-operative contrastenhanced magnetic resonance imaging and DTI fiber tractography for preoperative localization of important white matter tracts. Diffusion tensor imaging, by improving the recognition and characterization of white matter tracts, offers a glimpse into the brain microstructure at a scale that is not easily accessible with other modalities.

Results: The extent of white matter pathway involvement was clearly identified in all patients by using color-coded DT imaging maps and MR Tractography.

The study revealed that the most common tract involvement pattern detected was pattern (displacement). It was seen in 36 cases representing $72 \%$ of all cases. Pattern I (not affected) was detected in 6 cases representing 12\%. Pattern III (edematous) was detected in 11 cases representing $22 \%$. Pattern IV (partially disrupted) was detected in 13 cases representing $26 \%$. Pattern V (destroyed) was detected in 3 cases representing $6 \%$. Combined pattern of affection has been detected in most of the cases.

Conclusion: The effect of cerebral neoplasms on white matter pathways is not precisely understood with the aid of

Correspondence to: Dr. Samia Y. Heikal, The Department of Radio-diagnosis, National Cancer Institut, Cairo University standard diagnostic modalities. Diffusion-tensor imaging allowed for the preoperative identification of white matter pathways involved by tumor but the preoperative planning should be optimally complemented by various intraoperative procedures in order to insure a good neurological outcome of the surgery and valid functional prognosis.

Key Words: Diffusion tensor imaging( DTI) - Brain tumorMagnetic resonance image - Tractography White matter tracts.

\section{Introduction}

SURGICAL excision of brain tumors remains a big challenge especially in pediatric population. Brain tissue is delicate and vital and unlike most of human tissues, it does not regenerate. As all brain tissue is of crucial importance, there's no "safety margins" in brain tumor surgery. The neurosurgeon often finds himself in the dilemma of maximal excision versus maximal safety and reduction of postoperative morbidity caused by affecting eloquent areas in the brain. The most identifiable eloquent areas are the motor and sensory cortex, as well as their corticospinal and associated projections [1]. Improvement of the patient's outcome is primarily dependent on the preservation of cortical as well as subcortical function [2]

Traditionally, mapping of eloquent areas has been achieved by invasive methods such as direct electrical stimulation (DES) in the awake patient, implantation of a subdural grid with extraoperative stimulation mapping or operative sensory-evoked potential recordings. While accurate, these techniques are difficult to perform, place great stress on the patient and often require a larger craniotomy than necessary for the removal of the tumor [3] . 
DTI-FT represents a noninvasive technique for assessing tumor tissue characteristics in the preoperative surgical planning for malignant brain tumors as it gives information about white matter (WM) integrity of structures contiguous to the lesions before and after surgery providing prognostic data [4].Localizing the corticospinal tracts is especially critical in children because their white matter is maturing and, hence, is highly vulnerable to insult.

Various tumor-tract relationships can be appreciated by DTI including the identification of the tract predicted from its position and course and evaluating its proximity to the tumour as well as identifying the spatial position of the tumour in relation to the tract.

DTI parameters derive from the detection in vivo of the direction and the magnitude of water diffusion in biological tissues. The measurement of anisotropic diffusion of water molecules is calculated as the fractional anisotropy (FA) and represents a quantitative measure of WM microstructural integrity. A second parameter, which can be measured, is the apparent diffusion coefficient (ADC) which evaluates the overall magnitude of diffusion of water in tissue [4]

\section{Aim of work:}

The purpose of this study is to evaluate the role of MR diffusion tensor imaging and fiber tractography and its benefits over conventional MRI in accurate preoperative localization of important eloquent areas in pediatric brain tissue and their relation to the brain tumor.

\section{Patients and Methods}

\section{Patient population:}

This paper is adapted from the thesis submitted by the first author to the Faculty of Medicine, Cairo University, in partial fulfillment of the MD degree in Diagnostic Radiology. The work was a prospective study conducted on 50 pediatric patients with different types of brain tumors before undergoing surgery.

Males represented 26 patients $(52 \%)$ and females represented 24 patients (48\%) (Fig. 1).

The age of patients ranged between 1-16 years; mean age 7 .

The study was conducted at the Children's Cancer Hospital Medical imaging department in Cairo (57357) between June 2015 and April 2017.

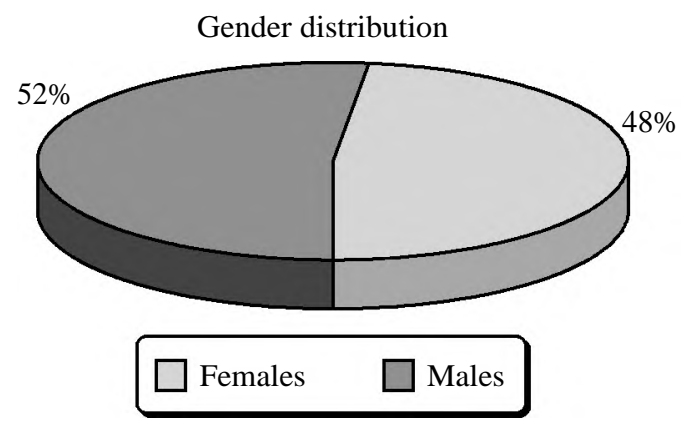

Fig. (1): Gender distribution of the patients included in the study, $26(52 \%)$ were males and $24(48 \%)$ were females.

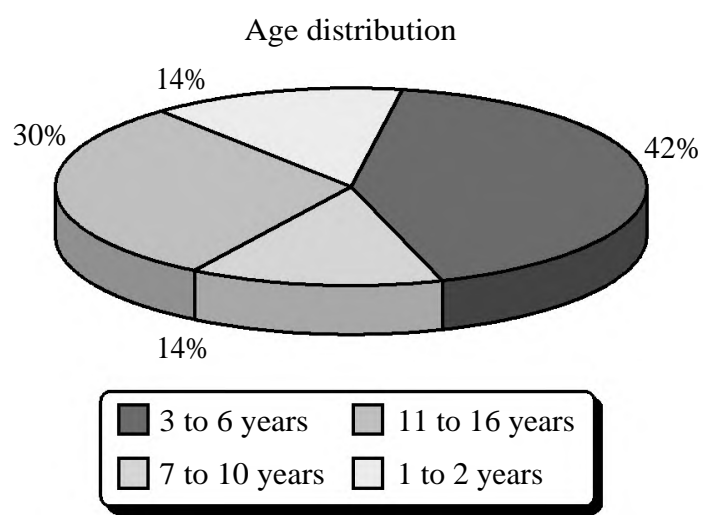

Fig. (2): Age distribution of the patients included in the study; 7 patients (14\%) ranged from 1 to 2 years (infant), 21 patients (42\%) ranged from 3 to 6 years (preschool), 7 patient (14\%) ranged from 7 to 10 years (primary) and 15 patients $(30 \%)$ ranged from 11 to 16 years.

Inclusion criteria included pediatric patients with brain tumours in their initial presentation. We included only primary lesions (except for one case of a single cerebral metastasis). Exclusion criteria included patients who received treatment before surgery, MRI contraindications or vitally unstable patients.

The study's protocol was approved by the Research Ethics Committee, Faculty of Medicine, Cairo University. Patients consents were unnesseccary as the DTI study was part of the MRI protocol for preoperative brain tumours.

\section{Methods:}

Each patient included in the study was subjected to full history taking.

Variable presentations were given by the patients with the most frequent neurological clinical presentation was headache followed by vomiting and visual symptoms. 


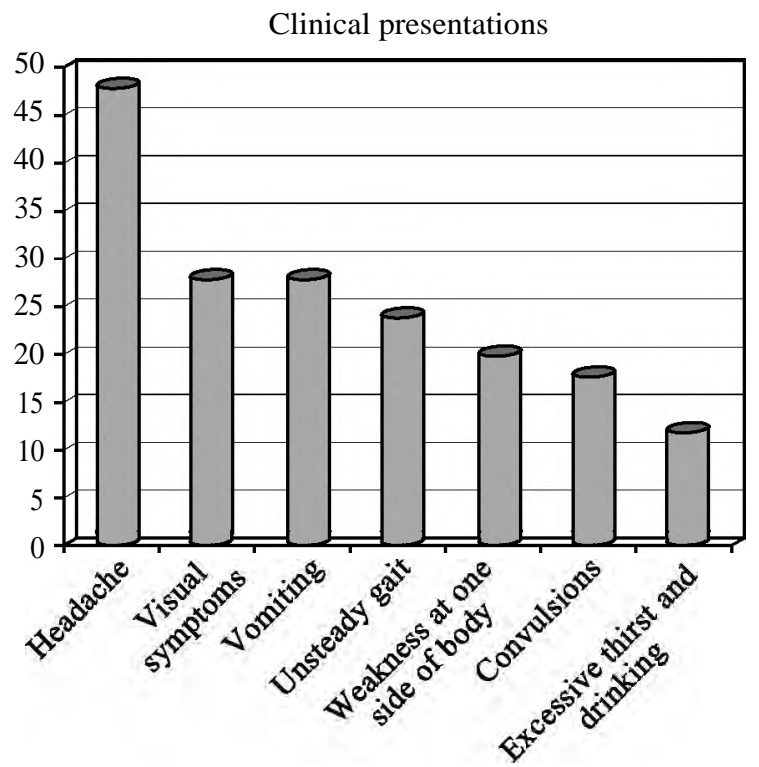

Fig. (3): Spectrum of clinical presentations of the study patient population.

Headache in 24 patients (48\%), vomiting in 14 patients (28\%), visual symptoms in 14 patients (28\%), unsteady gait in 12 patients (24\%), weakness at one side of body in 10 patients (20\%), convulsions in 9 patients $(18 \%)$ and excessive thirst and drinking in 6 patients $(12 \%)$.

\section{Magnetic resonance imaging protocol:}

MR examination Technique was performed using a 3 T Philips Ingenia scanner (Philips, Best, The Netherlands), software version 4. A multichannel head coil was used for the reception of MR signal.

Diffusion Tensor Imaging (DTI) data were obtained using single-shot spin echo, echo planar imaging sequence (TR/TE $8750 / 100 \mathrm{~ms}$ ) with parallel imaging (SENSitivity Encoding [SENSE] reduction factor of 2). Diffusion gradients were applied along 32 axes, using a $b$-value of 0 and $800 \mathrm{~s} / \mathrm{mm}^{2}$.

An acquisition data matrix of $112 \times 112$ was used, leading to voxel dimensions of $(2.5 \times 2.5 \times$ $3 \mathrm{~mm}^{3}$ ). FOV (field of vision) varied according to the patient's head size. Slices were acquired in the axial plane in a regular ascending order with a thickness of 3mm and no gap. A 3D T1-weighted image set (T1 $13 \mathrm{~W}-3 \mathrm{D}$ FFE (fast field echo), resolution $1 \times 1 \times 1 \mathrm{~mm}^{3}$ ) was acquired for anatomical localization. This was followed by axial 2D T2, T2 FLAIR and postcontrast T1 WIs for tumor characterization.

\section{Diffusion tensor imaging tractography:}

Image preprocessing and fiber tractography: The DTI and 3D T1 FFE images were transferred to the offline workstation (Philips View forumExtended MR Workspace version 3.2) where the DTI images undergo linear registration using the diffusion registration tool, to overcome the effect of Eddy currents and head motion on the diffusion data. Registered images are opened in the MR Diffusion application for production of parameteric maps (FA, FA color maps, ADC \& DWI trace). Registered Diffusion Tensor images were then loaded into the Fibertracking application, and the 3D T1 TFE images were used as the anatomical underlay for better localization of seed placement locations, color coded FA maps were viewed with anatomical images underneath giving both anatomical information and information about fiber orientation. The direction and anatomy of the tracts are seen in the directionally encoded FA maps, where a specific color is assigned to tracts running in the three orthogonal planes: red is for right to left tracts, green for anteroposterior tracts, and blue for craniocaudal tracts. A 3D display of tracts was created. For creating 3D fiber tracts, inclusionary ROIs (or seeds) was drawn (placed) along the course of the tract in the (axial, sagittal or coronal) color encoded FA map in single or consecutive sections, while exclusionary ROIs were drawn on the spurious fibers that needed to be excluded from a specific fiber bundle. The software then automatically traces the assigned tract and presents it in a 3D manner. Regions of interest (ROIs) were drawn within identifiable WM tracts affected by tumor, avoiding grossly cystic and necrotic regions, known fiber crossings, and gray matter. The following images were produced from the Fibertracking application:

$$
\begin{aligned}
& \text { 1- FA maps. } \\
& \text { 2- directionally-encoded color FA maps. } \\
& \text { 3- 2D fiber Tractography maps. } \\
& \text { 4- 3D fiber Tractography maps. }
\end{aligned}
$$

Color-coded DTI maps were analyzed, followed by tractography of individual tracts. The location of each tract and its hue on directional color maps were classified as normal or abnormal, based on comparison to the homologous tracts in the contralateral hemisphere, which were unaffected by tumor.

\section{We adopted the following suggested criteria:}

Pattern I (not affected) fibers are in the correct anatomical location, characterized by normal FA 
and normal ADC similar to the homologous tract in con-tralateral hemisphere.

Pattern II (Displacement): Was characterized by normal or mildly decreased FA and normal or mildly increased ADC relative to the homologous tract in contralateral hemisphere, with abnormal location and/or direction resulting from bulk mass displacement.

Patterns III (edematous): Characterized by decreased FA and increased ADC. The tract appeared in place or deviated on directional color maps with same colour but fainter due to decreased FA caused by the perilesional edema and the tract was seen passing through the high T2 oedema on conventional study.

Pattern IV (infiltrated and partially disrupted): characterized by substantially decreased FA and increased ADC. The infiltrated tract is in place or deviated on directional color maps with abnormal hues mostly attributed to disrupted fibers extending into different directions (spurious fibers).

Pattern V (destroyed): It was characterized by isotropic or near-isotropic diffusion, such that the tract or part of it was not identifiable on FA or dire- ctional color maps [6]

\section{Results}

Our study included different lesions of the brain, most common diagnosis were gliomas with their different grades.

According to the 2016 WHO grading system, the given pathologies are classified into four grades with each increasing grade implying lesser degrees of differentiation, increasing anaplasia, increasing proliferative potential, and mitotic activity.

The most common diagnosis were gliomas with their different grades. According to the 2016 WHO grading system [7], the given pathologies are classified into four grades with each increasing grade implying lesser degrees of differentiation, increasing anaplasia, increasing proliferative potential, and mitotic activity (Fig. 4).

- Grade I: Included 20 patients constituting 40\% of cases including DNT (Dysembryoblastic neuroepithelial tumours), craniopharyngomas, pilocytic astrocytomas, ganglioma and another low grade glioma.

- Grade II: Included 3 patients constituting $6 \%$ of cases including PXA (pleomorphic xanthoastrocytoma) and germinoma.
- Grade III: Included 10 cases constituting 20\% of cases including anaplastic astrocytomas, anaplastic epyndymomas, atypical neurocytoma and atypical pituitary adenoma.

- Grade IV: Included 12 cases constituting $24 \%$ of cases included medulloblastomas, glioblastomas multiforme, ATRT (Atypical Teratoid Rhabdoid tumour) and metastatic osteosarcoma.

- The pathology of five patients was not yet available at time of the study constituting $10 \%$ of cases.

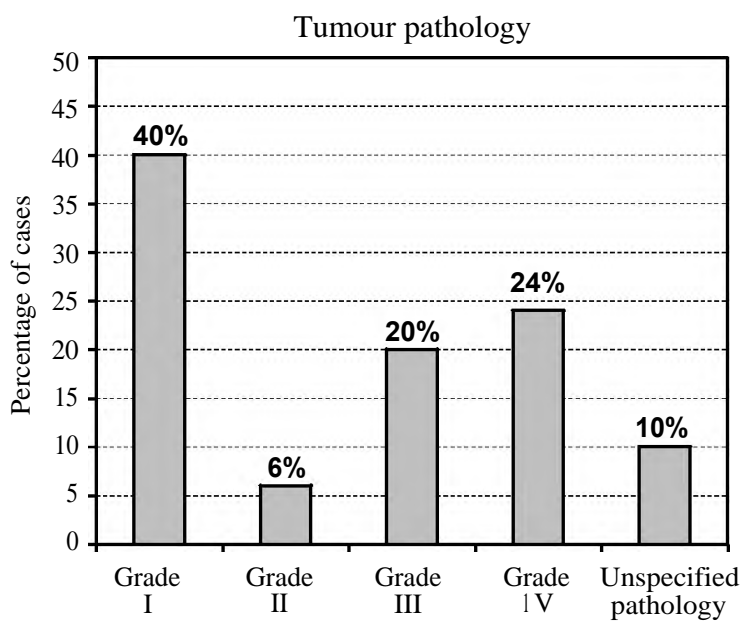

Fig. (4): Tumour classification included in our study.

We classified the tumors encountered in the study into two main groups, benign acting and malignant acting (Fig. 5).

1- Benign acting (grade I and II lesions) compromised 23 patients.

2- Malignant acting (grade III and IV lesions) compromised 22 patient.

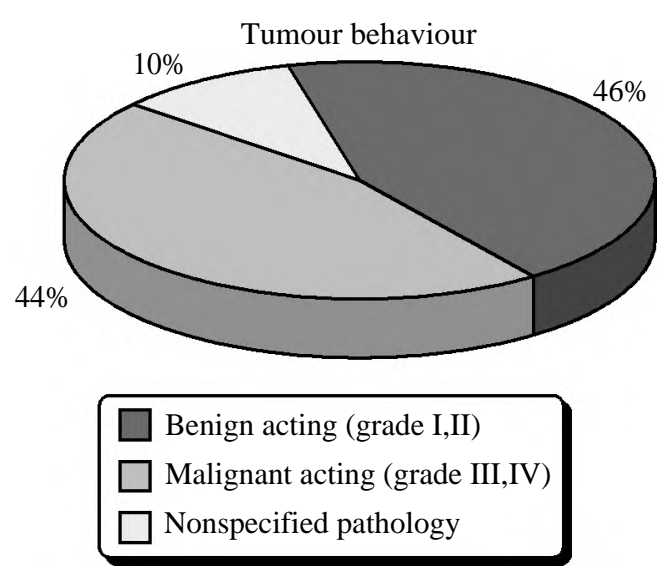

Fig. (5): Tumour behaviour (benign or malignant acting). 